\title{
SD-WDS: Bremsstrahlung Determination by Theoretical Calculation and Subsequent Estimation of Mass Attenuation Coefficients
}

\author{
Ken Moran ${ }^{1}$ and Richard Wuhrer ${ }^{2 *}$ \\ ${ }^{1 .}$ Moran Scientific Pty. Ltd, Bungonia, NSW, Australia. \\ 2. Western Sydney University Advanced Materials Characterisation Facility, NSW, Australia. \\ * Corresponding author: Richard.Wuhrer@westernsydney.edu.au
}

Over the years there has been some good work carried out on background corrections for quantitative analysis [1-4], in particular the mean atomic number (MAN) correction routine developed by Donovan and Tingle [4]. The MAN technique [4] allows for rapid calculation of the MAN background correction for wavelength dispersive spectrometry (WDS) data for most samples but has some limitations. The main factor limiting the MAN technique fully has been the WDS detector design utilizing a gas counter (GC). The traditional GC-WDS has very low energy resolution which means it is not really known what $\mathrm{X}$-rays are being counted by the detector, for example from secondary fluorescence and other X-rays (especially high order bremsstrahlung and other lines).

Most GC's have an order of magnitude poorer energy resolution (over $1000 \mathrm{eV}$ ) than the silicon drift detector (SDD), this means that if interferences are present you can get a corresponding improvement in background by removing them, either by energy discrimination, or physically removing their source. It is important to know whether they are there, so that you can remove them to improve your performance [5]. With the development of the high resolution silicon drift detector incorporated into the WDS (SDWDS) the artifacts usually counted by the GC-WDS can now be removed [5-8]. This now allows for the MAN technique to be improved for a more rapid background correction determination and subsequently more accurate analysis.

The superiority of a SD-WDS is easily seen once in operation [5-8]. The incorporation of the SDD has been found to improve the light and mid element range and consequently improving the detection limit for these elements. It is also possible to obtain more reliable results at high count rates with almost no change in resolution, gain and zero-peak characteristics of the energy spectrum [5-8].

It was found that data points that did not lie on the theoretical curve had some simple overlap or absorption issue, which can easily be resolved on further processing. In fact, bremsstrahlung study is a good way of characterizing absorption and has advantages compared to using characteristic lines. The major advantage is being able to obtain standards with a known concentration at the energy required (i.e. zero or much less than the detection limit (DL)). The next main requirement is counting statistics, which can be obtained by counting at least 40k counts at the energy required. Historically, bremsstrahlung has been represented by Kramer (1923) as [5]:

$$
\mathrm{Nc}(\mathrm{E})=a \mathrm{Z}\left(\mathrm{E}_{\mathrm{o}}-\mathrm{E}\right) / \mathrm{E}
$$

Where, $\mathrm{Nc}(\mathrm{E})$ is the intensity in photons per second per unit energy per incident electron, $a$ is a constant, $\mathrm{Z}$ is atomic number, $\mathrm{E}_{\mathrm{o}}$ is the initial energy of the electron and $\mathrm{E}$ is the energy of the excited photon. There are other more accurate modifications to Kramer's equation [5], but for simplicity equation 1 is suitable for energies above $2 \mathrm{keV}$. In this case, bremsstrahlung is dominated by the term $\left(\mathrm{E}_{0}-\mathrm{E}\right) / \mathrm{E}$ with all other terms constant for any given sample. For the study of bremsstrahlung generated within the 
sample, fixed energy values were observed and one atomic number $(Z)$ was ratioed to all others. Kramers's equation can be simplified then to:

$$
\mathrm{IBa} / \mathrm{IBb}=\mathrm{C} * \mathrm{Za} / \mathrm{Zb}
$$

Where IB is the Bremsstrahlung intensity generated, of element a or b, $\mathrm{C}$ is a constant for each energy value which is dependent on E, Eo and the efficiency of the detector.

Figures $1 \mathrm{a}$ and $1 \mathrm{~b}$ (red line) reveals a linear relationship between bremsstrahlung intensity and atomic number. The intensity given by this straight line is now corrected for absorption, continuum fluorescence and BSE losses to see the true value of the bremsstrahlung arriving at the detector. Figure 1a shows for high energy bremsstrahlung the corrections are moderate and nearly give a straight line. The blue points are measured from standards and green points are theoretically calculated.

For low energy bremsstrahlung, Figure $1 \mathrm{~b}$, there is a much stronger absorption effect. Blue and green points are as above. Absorption is calculated using a slightly modified Philibert expression and Heinrich's mass attenuation coefficients (MAC's) [2]. Absorption is the key to getting the bremsstrahlung and $\mathrm{Z}$ relationship correct. For elements below Fluorine there are few good sources of MAC's. The MAC's for $\mathrm{C}, \mathrm{N}$ and $\mathrm{O}$ are reasonable up to $\mathrm{Z}=30$ (also surface contamination can drastically absorb low energy radiation). Absorption of bremsstrahlung by $\mathrm{M}$ lines is another problem. On the positive side, it turns out that bremsstrahlung is a very good way to estimate MAC's [9]. This is currently being investigated.

References:

[1] HA Kramers, Philosophical Magazine 46 (1923), p. 836.

[2] KFJ Heinrich, X-ray Optics and Micronanalysis, Ontario, Uni, of of Western Ontario (1987) p. 67.

[3] JT Armstrong in "Electron Probe Quantitation", Heinrich, K.F. (Plenum Press, New York) p. 261.

[4] JJ Donovans and TN Tingle, Journal of Microscopy Society of America 2 (1) (1996), p. 1.

[5] R Wuhrer and K Moran, IOP Conf. Series: Materials Science and Engineering 304 (2017).

[6] K Moran and R Wuhrer, Microsc. Microanal. 22 (S3) (2016), p. 92.

[7] R Wuhrer and K Moran, Microsc. Microanal. 23 (S1) (2017), p. 1048.

[8] K Moran and R Wuhrer, Microsc. Microanal. 24 (S1) (2018), p. 756.

[9] K Moran and R Wuhrer, Silicon Drift Detector Incorporated into Wavelength Dispersive Spectrometer (SD-WDS) - Allowing Bremsstrahlung Determination by Theoretical Calculation, EMAS (2019), Thondheim Norway.

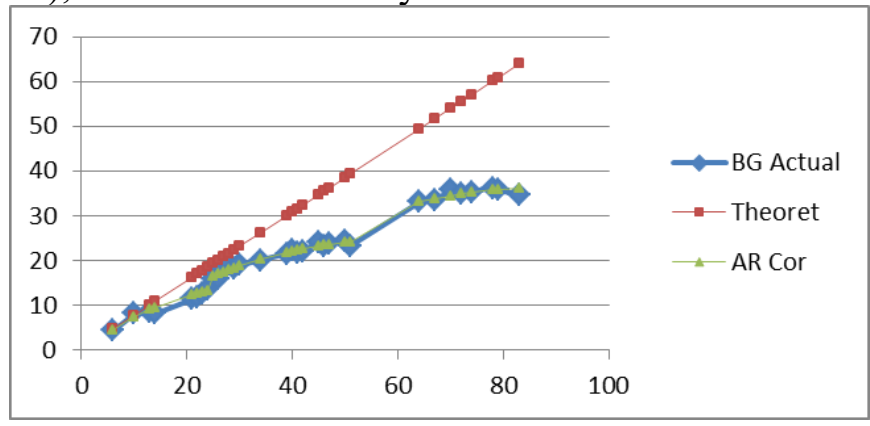

a.

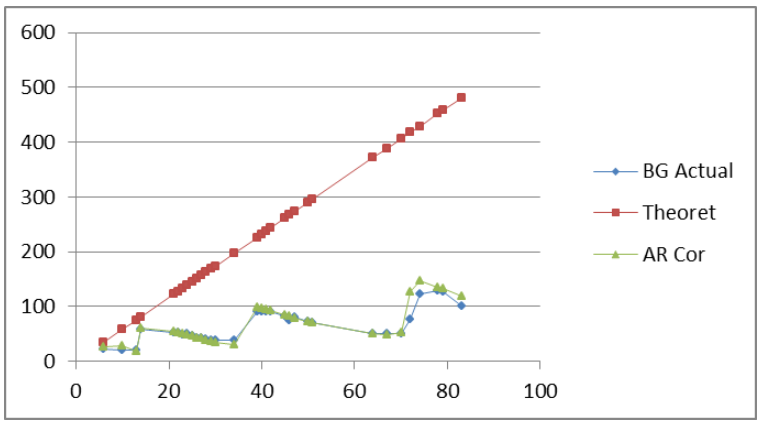

b.

Figure 1. a) Intensity (cps/nA on LIF) vertical axis and Atomic number (Z) on horizontal axis measured at $6.4 \mathrm{keV}$ for (Fe) and b) Intensity (cps/nA on TAP) versus $\mathrm{Z}$ measured at $1.74 \mathrm{keV}$ for ( $\mathrm{Si}$ ) [9]. 